\title{
Pervasive Electricity Distribution System
}

\author{
Muhammad Usman Tahir, Mohammad Asif Khan, Ahmad Waqas, Shahneel \\ Siddiqui \\ Department of Electrical Engineering, Sukkur Institute of Business Administration \\ Sukkur, Pakistan \\ usman.ee@iba-suk.edu.pk, asif.khan@iba-suk.edu.pk, shahneel.ee@iba-suk.edu.pk, \\ ahmad.waqas@iba-suk.edu.pk
}

\begin{abstract}
Now a days a country cannot become economically strong until and unless it has enough electrical power to fulfil industrial and domestic needs. Electrical power being the pillar of any country's economy, needs to be used in an efficient way. The same step is taken here by proposing a new system for energy distribution from substation to consumer houses, also it monitors the consumer consumption and record data. Unlike traditional manual Electrical systems, pervasive electricity distribution system (PEDS) introduces a fresh perspective to monitor the feeder line status at distribution and consumer level. In this system an effort is taken to address the issues of electricity theft, manual billing, online monitoring of electrical distribution system and automatic control of electrical distribution points. The project is designed using microcontroller and different sensors, its GUI is designed in Labview software.
\end{abstract}

Keywords: component; Pervasive, Smart Grid, Power Distribution

\section{Introduction}

Currently Pakistan is suffering from severe energy crisis. Energy is part and parcel of every aspect of life as well as backbone of a country's economy. Pakistan is lagging behind in this area despite rich reserves of raw energy, still the country is struggling to fulfil its energy needs. There is a dire need for the investment and proper use of these reserves for driving the wheel of economy. Steps must be taken to eliminate the electricity theft around the country and minimize the usage of electricity to save our future generations from facing further energy crisis [1].

In this project a system has been developed to reduce the energy consumption around the country and eliminate the theft of electricity which is happening in different areas of Pakistan. The project is based on specifically three parts: substation, distribution and the consumer device unit. Substation works as the main control center which has total authority to allow and disallow any connection to any consumer, and also has the ability to detect any theft. The substation also monitors the usage of electricity provided to consumers and the feeder lines. The Distribution works as the current sensor calculates the flow of Power through the Electrical cables that are used to distribute power to the consumer houses, from the sensor system sends the data to the substation via Cellular Network. The Consumer device works as the Smart Energy Meter. There is also a touch screen panel for consumers that 
provides various functions to the consumer which include monitoring of the energy consumed by the load and controlling the usage of electricity by setting a limit of Energy Units to be consumed by the load. Implementation of this project will reduce the over usage of electricity by the consumers, and will provide an interface to the users through which they can monitor their usage of electricity. The system will also detect electricity theft, if occurred, and will alert the substation instantaneously. The system aims to eliminate the settlement between the Meter Readers and the consumers, as the system will end the role of
Section IV is about the different results taken during experimentations of the project. Section $\mathrm{V}$ will tell you about the different work carried out to solve the said problem.

\section{Existing Manual Electricity Distribution System}

The energy infrastructure of Pakistan is not up to date and well-maintained, rather it is considered to be underdeveloped and poorly managed. Currently the country is facing severe energy crisis. Despite strong economic growth and rising energy demand during the past decade, no serious efforts have been made

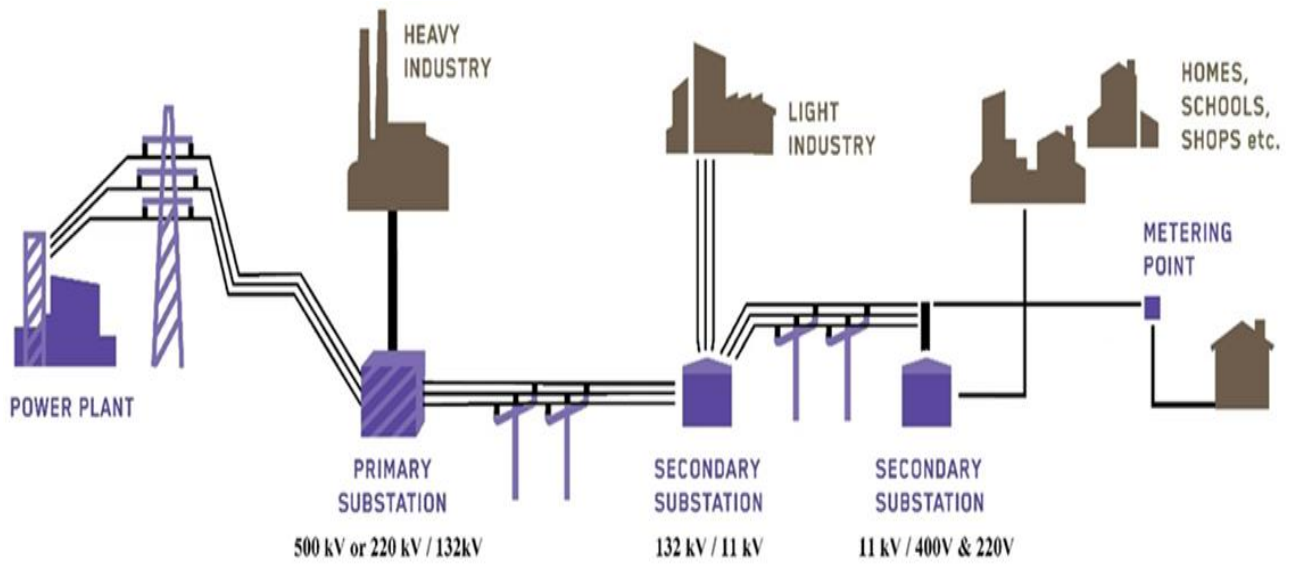

Figure. 1: Exiting Electrical Distribution System

meter readers to manually read the Energy Units consumed by each customer. Successful implementation of this system around the country will reduce the energy consumption by a good rate, in turn decreasing the rate of electricity load-shedding in Pakistan making the lives of people happier and better.

The paper is divided into five sections. Section II is about the existing electrical infrastructure which is present in Pakistan for the distribution of electrical power to homes and industry. Section III is about the PEDS, its architecture, different parts of the project and working principle. to install new power generation plants. On the other hand demand for electrical power is increasing day by day.

The rapid demand growth, transmission losses due to outdated infrastructure, power theft, and seasonal reductions in the availability of hydropower have worsened the situation. Consequently, the demand exceeds supply and hence loadshedding is a common phenomenon through power shutdown. In the existing system the power is being distributed to primary substation which is being step up by the station to $500 \mathrm{kv}$ or $220 / 132 \mathrm{kv}$ and transmitted 
on transmission lines of long distances. The $500 \mathrm{kv}$ is then being received at secondary substation which steps it down to $11 \mathrm{kv}$ for transmitting it to shorter distances to the distribution transformers where it is stepped down to $400 \mathrm{v}$ and $220 \mathrm{v}$ for domestic usage. Currently there is no system in Pakistan to monitor the transmission of power from secondary substation to transformer point and from transformer to consumers. Most of the connection and maintenance is done manually. The connectivity of the existing electrical transmission and distribution system is show in Fig.1.

To improve the existing system various incentive rate design have been implemented in the past to improve the power transmission process and to minimize the line losses [2]. Power transformation from power plant to substation is reasonable but from substation to consumer level it is going worst. This happens due to poor monitoring system, manual metering devices, human interaction and electricity theft. In traditional electricity distribution systems, metering is done only at one point consumer level. There is no care about in and out measurement of power at distribution transformer as shown in Fig.1. The main limitations of the existing system are electricity theft, manual operations, fault rectification, manual billing and highly human dependent system.

\section{Pervasive Electricity Distribution System}

Pervasive electricity distribution system (PEDS) aims to decrease the theft, corruptions, and extra usage of electricity by the consumers and would bring transparency in the distribution, hence decreasing the shortfall of electricity around the globe and Pakistan. The system is designed to replace the old power distribution system.

\subsection{Main Features of the System}

The main purpose of this system is to design a smart electricity distribution system which can eliminate electricity theft and reduce the energy consumption and provide an interface to the customer which in turn would help him to monitor and control the usage of electricity. This system would also serve:

- To eliminate the need of manual meter readers to read the units of energy consumption

- To smartly calculate the energy units of all houses

- To alert the distribution unit, if any electricity theft is occurred

- To implement a real time communication between substation and distribution units through a GSM network

- To provide a smart interface to the user, which can help him to monitor and control the electricity usage via a touch screen module

\subsection{System Architecture}

The system is mainly consisting of three major parts: the substation unit, distribution unit and consumer device unit. The substation unit mainly consists of a computer on which GUI will be provided for controlling distribution and collection of data. The system will be able to communicate wirelessly via cellular network. The substation unit maintains a database of every user which contains the information about the energy usage of every customer from time to time and produces a monthly record of units consumed in the form of a bill. The substation unit is managed by the electricity providers.

The substation has a control over all of its customers, and has the authority to allow or terminate the connection of any customer at any time, and it also houses an indicator to detect any occurring electricity theft in the area. The substation unit also produces graph of power being consumed of each customer 
and calculates the power at the distribution and matches it with the power readings that it
The consumer device unit performs various functions. It is basically a touch screen LCD

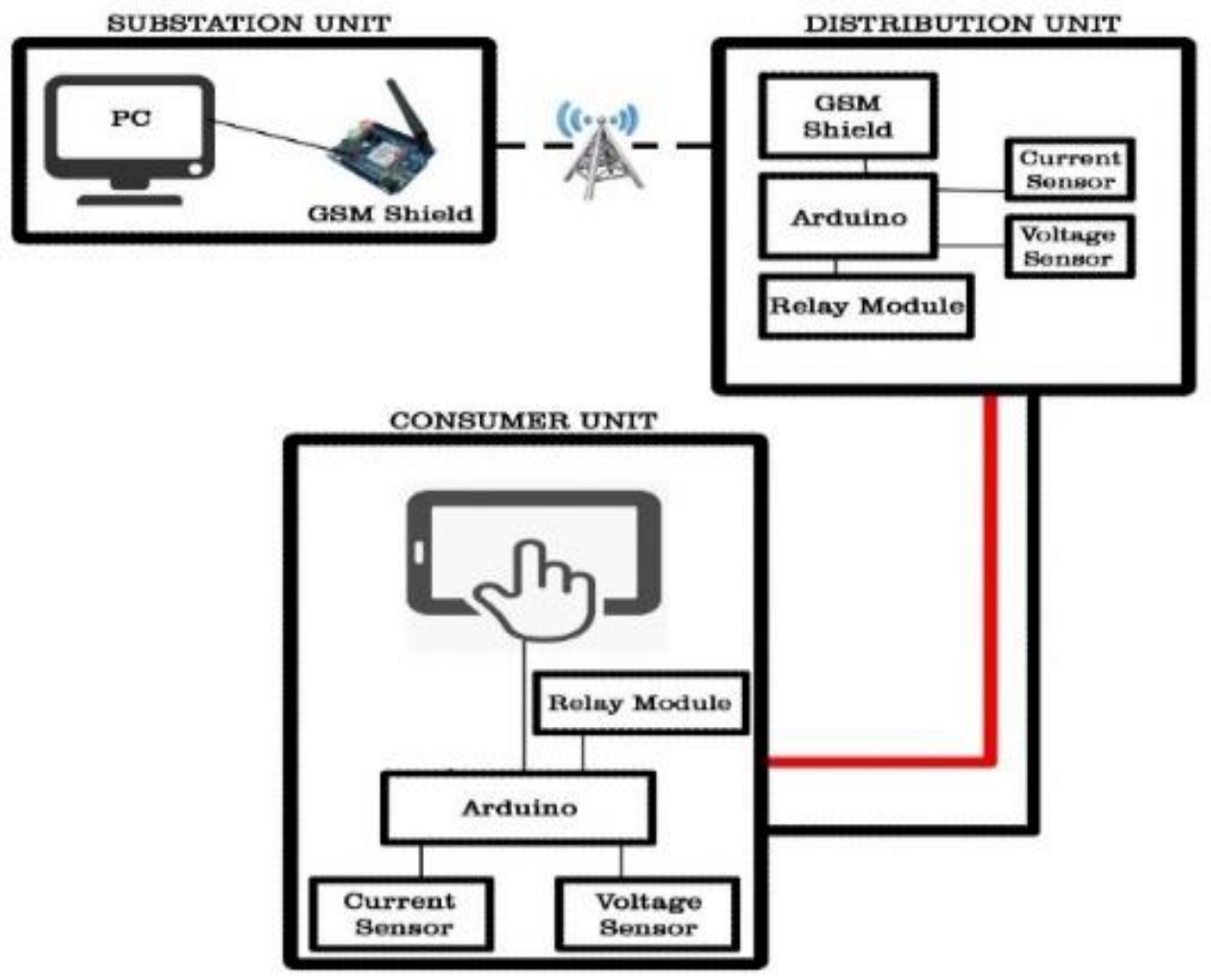

\section{Figure. 2: PEDS System Architecture}

gets via cellular network from consumer devices. If no match occurs, the theft seems to have occurred.

The distribution unit is installed at distribution transformer from where electricity connection is provided to every user. This unit has microcontroller, current and voltage sensors, relay and cellular GSM shield for wireless communication. It senses the power that flows through the lines to the users and calculates the total power that flows before it is being distributed to the consumer, and keeps sending the data to the substation for monitoring and maintenance purpose [3]. that is interfaced via Arduino and allows the user to control the usage of load and monitor the energy usage of the load. The consumer device of the project holds an option for the consumer to monitor its energy usage, where the consumer would be able to view the energy readings including the voltage reading, current reading, and the multiplication of both, that is, power reading. The user can also view a graph power vs. time of the load. The consumer device of the project serves the customer to control his usage of electricity by setting a limit of energy usage of the house. Once the energy limit is set to a particular value, then the load will be disconnected from 
the supply of electricity as soon as the power consumed has reached up to the value of the energy limit. The complete system model is depicted in Fig. 2.

\subsection{GUI development in LabView}

In PEDS, there is a strong role of control interface at substation. This system is designed in LabView with user friendly GUI. The main program or server program contains multiple Windows. Home window is the first window on server for selection of a particular feeder. By selecting a particular feeder, another window appears for selection of a particular user in that feeder. In this window, there is a list of many users in that feeder. When we select a particular user, we can see user description, history of bills and a textbox to send text to a particular user.

This is the main window which appears at PC on which LabView is installed in substation unit [4]. This window provides a selection menu for feeder lines which are Feeder Lines, Users, Energy Ratings, History, Termination Option and Theft Detection, as shown in Fig. 3. Each option is having its own window and multiple functions for the remote monitoring and controlling of the data which

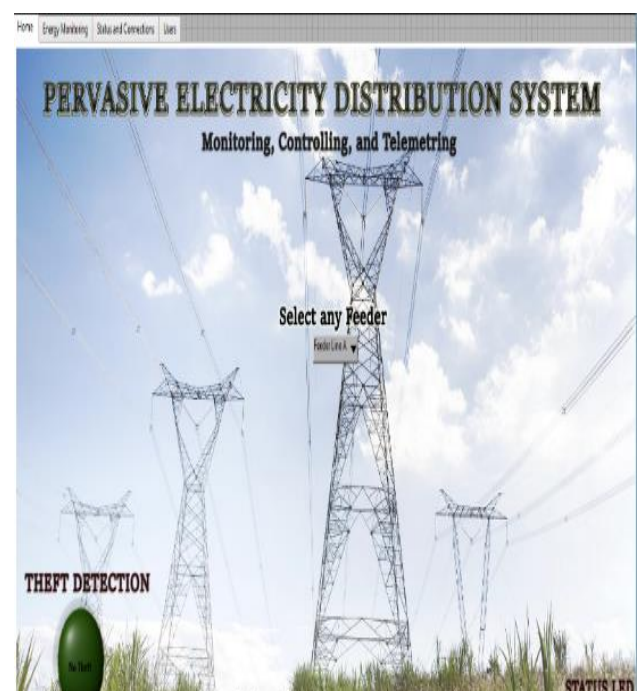

Figure. 3: Substation GUI window is gathered from remote devices installed at distribution unit and consumer devices.

\subsection{Consumer Device Unit of the Project}

The consumer device unit of this project is to be fitted inside the houses of the consumers. It incorporates a touch screen panel that provides a graphical user interface to the consumers to use various functions shown in Fig. 4, like to see energy

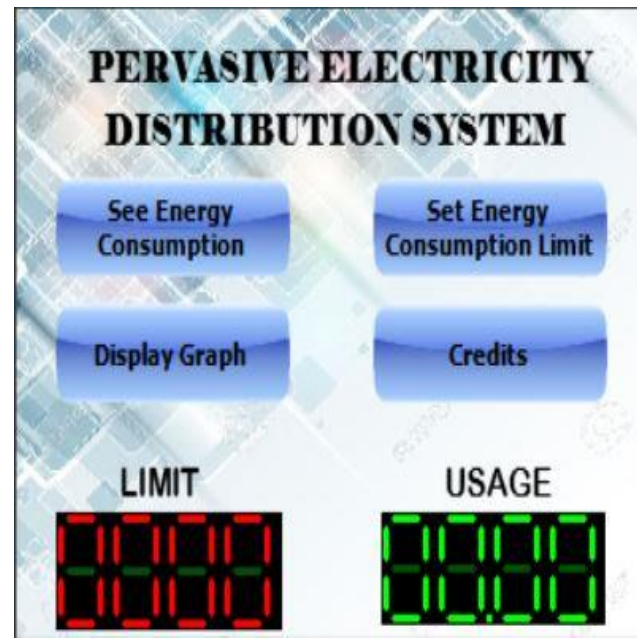

Figure. 4: Consumer Device GUI

consumption, set energy consumption limit, display consumption graph and display credits.

\section{Results}

The prototype of the PEDS was developed and results were gathered to test the novelty of the system. Real-time test of the system was done by connecting an electrical load to it. The main purpose was to feed data to the system in the real-time manner, also to detect electricity theft and remote control from substation GUI. Table 1 shows reading regarding theft detection. Current sensor 1 measures the current of load one, current sensor 2 measures the current of load two and current main line is the current measured at transformer via substation unit. The sum of 
currents of individual users is compared with total current, unbalanced results depict theft detection. In the last row of the table shows imbalance in current. The sum of currents of individual user is 4 amperes, whereas total current at transformer is 5 amperes, it means 1 amperes of current is theft and also same is the case in the third row.

Table 1: Theft Detection

\begin{tabular}{|c|c|c|c|c|}
\hline $\begin{array}{c}\text { S. } \\
\text { No } \\
\cdot\end{array}$ & $\begin{array}{c}\text { Current } \\
\text { at User } \\
\mathrm{A}\end{array}$ & $\begin{array}{c}\text { Curren } \\
\mathrm{t} \text { at } \\
\text { User B }\end{array}$ & $\begin{array}{c}\text { Current } \\
\text { at } \\
\text { Distributi } \\
\text { on }\end{array}$ & $\begin{array}{c}\text { Theft } \\
\text { Detection } \\
\text { LED }\end{array}$ \\
\hline 01 & $1 \mathrm{~A}$ & $1 \mathrm{~A}$ & $2 \mathrm{~A}$ & OFF \\
\hline 02 & $2 \mathrm{~A}$ & $1 \mathrm{~A}$ & $3 \mathrm{~A}$ & OFF \\
\hline 03 & $1 \mathrm{~A}$ & $2 \mathrm{~A}$ & $4 \mathrm{~A}$ & ON \\
\hline 04 & $2 \mathrm{~A}$ & $2 \mathrm{~A}$ & $4 \mathrm{~A}$ & OFF \\
\hline 05 & $2 \mathrm{~A}$ & $2 \mathrm{~A}$ & $5 \mathrm{~A}$ & ON \\
\hline
\end{tabular}

Electricity theft can be detected easily in any feeder line at substation by the software`s home window so that company can take action. The software designed in LabView maintains the Database of every use in excel file as shown in the figure of User B displaying the power consumption of each user at every instant of time as it is illustrated in the Fig. 5 and Fig. 6.

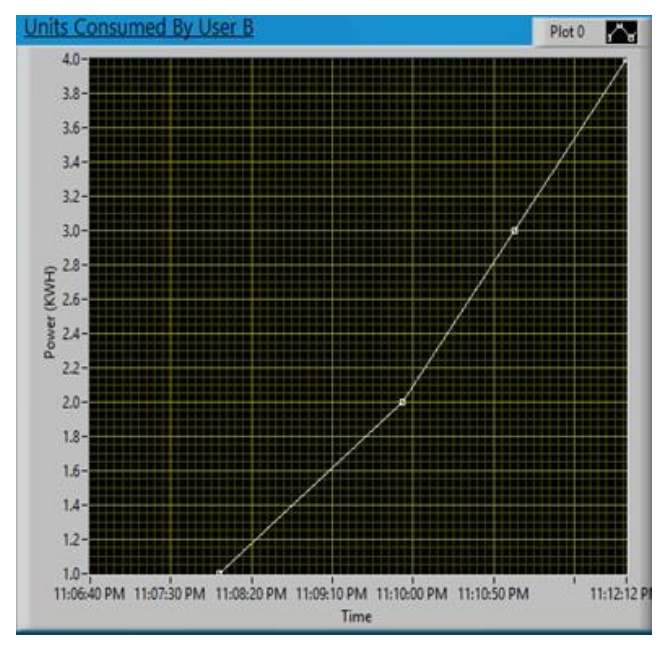

Figure. 5: User "A" Real-time Consumption
The system is capable of maintaining the realtime consumption of every user which is connected to the distribution transformer. The graph is plotted between the $\mathrm{kWh}$ and the current time.

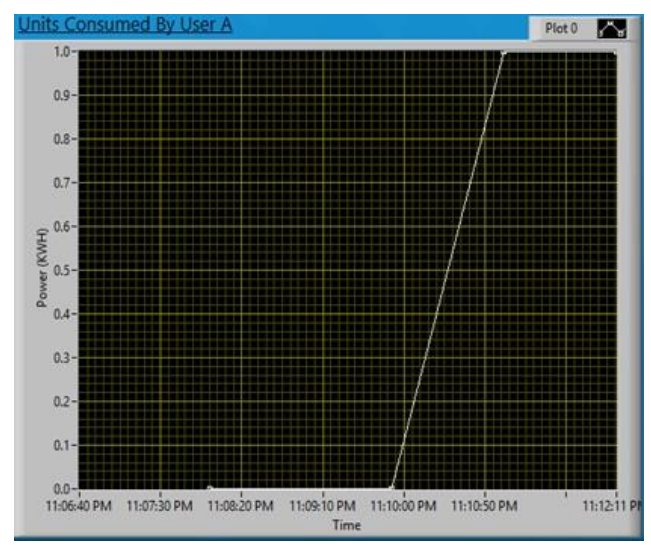

Figure. 6: User "B” Real-Time Consumption

\section{Related Work}

The importance of electrical power has forced the research world to do some good work to make electrical systems more reliable, efficient and affordable. In this regard, different researchers have worked on smart grid technologies. The background of the research includes Energy Management System, Demand Side Management, Load Management, Smart Grid, Pole Mounted Transformer Protection, Telemetry and Remote Monitoring and Management (RMM) [5]. Xuenan Gu et al. (2016) have worked on method for load transfer in case of any fault, their main target is to secure the important customer by providing power in case of fault [6]. Devidas, A.R et al. (2010) have worked on the development of smart system for controlling power transmission faults, power theft by using sensors nodes at various power points to monitor the power and act accordingly [7]. Habib Elkhorchani et .al. (2010) are working on the communication 
modeling between the different parts of a smart grid system [8]. Neal Master et al. (2014) have worked on the decentralized modeling of smart by using different algorithms [9].

\section{Conclusion and Future Work}

In this project of Pervasive Electricity Distribution System the work has been done to overcome major issues with current existing outdated energy transmission infrastructure of the country. Currently the main issues of the current energy crises are the electricity theft done by consumers, wrong meter reading and waste of human resources over meter reading, losses during transmission, manual electrical grid working. Also the usage of electricity at homes is not efficient or optimized. This project has been developed by keeping in view the above issues. With this project theft can be reduced by automatic meter reading system, also substations can be automated to have remote control of consumers' connection and their status.

The future expansion of this project will be divided into two parts: one for consumers and other for distribution and substation units. Machine learning can be used to find the patterns of user usage and to automate switching accordingly. The second part of the project needs fine tuning for testing it in real electrical distribution and substation units.

\section{References}

[1] Budsock, A., "Affordable and Clean Energy for All - Youth Lighting up the World." Energy Overview. The World Bank, 05 Apr. 2016. Web. 21 June 2016

[2] Ehrhardt, K.M.; Laitner, S., "People Centered Initiatives for Increasing Energy Savings," 2010.

[3] Lunsford, J.M., "Overcurrent protection of pole top distribution transformers that have tank mounted lightning arresters," in Transmission and
Distribution Conference, 1996. Proceedings, 1996 IEEE, vol., no., pp.586-590, 15-20 Sep 1996.

[4] Higa, M.L; Tawy, D.M.; Lord, S.M., “An introduction to LabVIEW exercise for an electronics class" Frontiers in Education, 2002. FIE 2002. 32nd Annual (Volume: 1) 2002

[5] Robert Searle, "Demand Management." Office of Energy. Government of Western Australia, n.d. Web. 30 Nov. 2010.

[6] Xuenan Gu ; Xingying Chen ; Kun Yu, " Research on the load transfer strategy of smart distribution grid considering the important power customers," in Power and Energy Engineering Conference (APPEEC), 2016 IEEE PES AsiaPacific

[7] Devidas, A.R.; Ramesh, M.V., "Wireless Smart Grid Design for Monitoring and Optimizing Electric Transmission in India," in Sensor Technologies and Applications (SENSORCOMM), 2010 Fourth International Conference on, vol., no., pp.637-640, 18-25 July 2010.

[8] Habib Elkhorchani ; Khaled Grayaa, " Smart micro Grid power with wireless communication architecture," in Electrical Sciences and Technologies in Maghreb (CISTEM), 2014 International Conference on, 3-6 Nov. 2014

[9] Neal Master ; Jeffrey Mounzer ; Nicholas Bambos, "Distributed smart grid architecture for delay and price sensitive power management", Communications (ICC), 2014 IEEE International Conference on, 28 August 2014 